\title{
The COVID-19 Pandemic
}

\author{
Jadab Chandra Chattopadhyay \\ Professor and Head of Department (Anatomy), Medical College Kolkata, West Bengal, India.
}

\begin{abstract}
Recent COVID-19 pandemic has caused the greatest crisis in human civilisation worldwide. Initially started in Wuhan, China it has gradually spread all over the world due to the time taken by the people to understand the seriousness of the disease. Initial symptoms are unremarkable and typical of upper respiratory tract viral infection, followed by dry unproductive coughing, low grade fever and ultimately full blown feature of pneumonia requiring hospitalisation and respiratory assistance including ICU and ventilation. Older adults, immunocompromised patients and those with several co-morbidities fall in higher risk categories irrespective of age. In this paper the origin, spread of infection and virulence, preventive steps, diagnosis and recent trends in clinical management is discussed in detail. Future implications on socio-economic point of view is mentioned in global as well as in national perspective.
\end{abstract}

Key words: COVID-19, SARS-CoV, RT-PCR,G-6-PD, Quarantine.

\section{Introduction}

The greatest crisis human civilization has ever faced in recent days is the toll of COVID-19 worldwide. The brunt of attack is fiercer than that of World War I\&II. The infection of this contagious viral disease is more than the wildfire spread. This has haunted the sleep of human race. A single stranded RNA virus with a capsidcover is declaring war against multicellular organisms like human being having endless number of DNA and RNA.

Our planet earth is the abode of all from microbes to multicellular Homo sapiens. Louis Pasteur postulated that rabies was caused by a living thing smaller than bacteria and proposed the term virus (Latin: poison). Viruses are noncellular parasites with definite size, shape and chemical composition and can be cultured in laboratory. They can infect every type of cell like bacteria, algae, fungi, protozoa, plants and animals including humans. Viruses can infect animals and in turn humans causing zoonosis. Ultramicroscopic in size (20 $\mathrm{nm}$ to $450 \mathrm{~nm}$ ), geometric in form ,consist of protein capsid covering nucleic acid RNA or DNA, viruses are inert outside host cells and multiply by assembly line method but not by dividing. They are obligate intracellular parasites of host cells. Viruses can cause infectious diseases, birth defects, cancers in animals including humans and cultivated plant infections with adverse agro-cultural, economic and ecological repercussions and used as invaluable tools to study basic genetic principles and cytopathic effects.

\section{Nature, Origin, Spread, Infection and Virulence}

The name 'Coronavirus' is derived from the Latin word corona meaning crown or halo which refers to the characteristic appearance of peplomer spikes around the virions giving appearance of a solar corona when viewed under

\footnotetext{
* Address of Correspondence: Prof. Dr. Jadab Chandra Chattopadhyay.

Affiliation: Professor and Head of the Department (Anatomy), Medical College Kolkata, West Bengal, India. Email address: drjadabchatterjee93@gmail.com
} 
two dimensional transmission electron microscopy. They are large spherical viruses having a helical symmetry. They possess linear positive sense single stranded RNA of 26 to $32 \mathrm{kbp}$ size, largest known genome of all RNA viruses. The genomic RNA is capped and polydenylated and comprises several genes encoding both structural and non-structural proteins (1). CoV replicates in the cytoplasm and bud off the cytoplasmic membrane. Virion release occurs via membrane fusion and exocytosis with eventual cell death. CoV has a very high mutation rate that has led to altered properties, host ranges and disease manifestations. There are six recognized coronavirus that are known to cause human infection, mostly belonging to beta coronavirus group.

Recent classification: 1) Human coronavirus 229E 2) Human coronavirus NL63 3) Human coronavirus OC43 4) Human coronaviruses HKUI 5) SARS CoV 6) MERS-CoV. In 2019-20 epidemic, the causative agent is a novel virus belonging to family coronaviridae, named 'COVID-19', an acronym of coronavirus disease 2019. Many CoV has been identified in a variety of different animals like mice, rats, chickens, turkeys, swine, dogs, cats, rabbits, cattle and humans and cause a variety of diseases like gastroenteritis and respiratory tract disease.

The COVID-19 particle is heavy and gets grounded over surface very soon. Rarely it may be air-borne in favourable situations. SARS-CoV, mainly zoonotic in origin have crossed into human most likely from animal host like bats and pangolins in the wild game markets in Guangdong, China. The early transmission of SARS-CoV was inefficient causing little disease but later it adapted towards greater fitness for efficient human to human transmission. Major routes of $\mathrm{CoV}$ transmission in humans are droplet infection, aerosolisation and fomites. Deposition of droplets onto the respiratory epithelium irritates it. Droplets spread by coughing, sneezing and close personal contacts, touching mouth, nose, and eyes or shaking hands. During SARS$\mathrm{CoV}$ outbreak, infected patients disproportionately contributed to widespread transmission to hospital staff members.

Number of infected hospital staff members are correlated with: 1) Number of infected patients 2) Length and type of exposure 3) Use of Personal Protective Equipment (PPE) 4) Use of preventive measures $(2,3)$. Other factors determining transmission are co-infection with other viruses and immunosuppression. People who are at higher risk for severe COVID-19 illness include older adults ( $<=65$ years), people who live in a nursing home or long-term care facility. In addition people with chronic lineage disease or moderate to severe asthma, heart conditions, those who are immunocompromised (on cancer treatment, immuno-deficiences, smokers, AIDS affected, prolonged user of steroids or over immune weaking medications), people with severe obesity (BMI of 40 or higher), diabetes, with chronic kidney disease undergoing dialysis or having liver disease fall under higher risk categories irrespective of their age.

Initial symptoms are typical of an upper respiratory tract viral infection. After a few days, coughing and low grade fever progress rapidly into full blown pneumonia requiring hospitalisation and often mechanical ventilation. Fever, malaise, lymphopenia, elevated liver enzymes, together with infiltrates and consolidation upon chest $\mathrm{x}$-ray. Illness ranges from mild symptoms to severe illness and death. These symptoms may appear 2-14 days after exposure (based on incubation period). Fever, dry cough, shortness of breath. Emergency warning signs include trouble in breathing, persistent pain or pressure in the chest, inability to arouse, bluish lips or face (cyanosis) and profound weakness. Complications are manifested in full blown pneumonia requiring hospitalisation, mechanical ventilation and critical care managements.

Initially started at the Wuhan of China and spread all over the globe affecting Italy, Spain, France, UK ,USA, Korea, Iran and Asian countries like India. Spread in India is due to the time taken by the people to understand the seriousness of COVID-19 spread worldwide. The virus is highly infectious, spreads quickly from contact to produce the new cases through droplets, aerosols, fomites. Infectivity transmission is through hand to hand, touching mouth, nose and eyes. Infection spreads to throat, upper respiratory tract. Upper myocarditis is common leading to heart failure in senior citizens. Infectivity is quite high but the organism is less virulent 
demanding on an average between $2-3 \%$ if proper healthcare delivery system is adequate. Virulence is raised high by presence of comorbidities like COPD, Myocardial Infarction, CRF and ageing.

\section{Clinical Findings}

Initial symptoms are unremarkable and typical of upper respiratory tract viral infection, followed by dry unproductive coughing, low grade fever and ultimately full blown feature of pneumonia requiring hospitalisation and respiratory assistance including ICU and ventilation. Features are fever, malaise, hypothermia, lymphopenia and elevated liver enzymes. X-ray shows infiltration and consolidation, quantitative PCR studies for viral RNA and rapid test kits for antiviral antibodies are used and shows viral load. RT-PCR (Reverse Transcriptase PCR) is sometimes helpful. Other findings are peripheral Tcell lymphocytopenia, and reduced CD4 and CD8 cell counts (3). Myocarditis with imminent cardiac failure is common. Multiorgan affection is common and variable.

\section{Preventive Measures}

Coronavirus is large in size (400-500 micron), heavy and grounded, not transmitted easily by air distally. Masks prevent its entry. Handwashing with soap water and alcohol based sanitizer kills it. The aim of prevention is to break the transmission chain of the virus. It spreads by hand to hand and foot. Virus spreads by touch and entry is mainly through mouth, oral cavity, nose and conjunctival sacs. To prevent these, an increase in social awareness of transmission, infectivity and virulence of the organism is required. For this reason, steps like nationwide 'janta' curfew and lockdown is undertaken to prevent community transmission (2).

Homestay and social distancing will reduce the new contacts and cases and will delay the transmission with flattening of the COVID-19 curve to the baseline. To avoid transmission to frontline health workers like doctors, nurses, paramedics and related staffs working in fever clinic, hospital quarantine, isolation, handling test kits and receiving samples of saliva, blood, nasopharyngeal swabs and broncho-alveolar lavage should use PPE (Personal Protective Equipment) like N-95 masks, COVID goggles, gloves, protective aprons, shoes with covers with caution. Training of donning and doffing of PPE has to be undertaken by them.

\section{Recent Trends in Management of Covid-19}

Usually patients come with symptoms of fever, upper RTI (Respiratory Tract Infection), weakness and body ache. In the fever clinic, detailed history, of travelling of patient or his/her family are taken and classification of patient done for Home quarantine, Hospital quarantine, Isolation or Critical care as the case may be. For presence of symptomatology in quarantine, tests are done for COVID-19 and if the test is positive, a patient is kept in isolation. During isolation, if there is an aggravation of symptoms, then they are sent to critical care (ICU).If the tests are negative, they are sent home after a period of 14 days in quarantine .Basics of treatment in quarantine is observation for symptoms of COVID-19 and testing (to the subjects with positive travel history or contact history with the travellers) as per ICMR guidelines (2).

Treatment given are mainly symptomatic and supportive. Prophylactic treatment with azithromycin or hydroxychloroquine (HCQ) (4) for positive cases and frontline health workers. Other treatments are anti-corona antivirals (5) with varied benefit and convalescent plasma. Prop up position, oxygen and ventilator support depending upon the Sp02\%.Treatment modalities are HCQ, Azithromycin, symptomatics, steroids, anti-viral substance in COVID19 , convalescent plasma, ventilatory support CPR with caution.

Azithromycin: This can be used to prevent upper RTI and sore throat at the beginning. In patients with hepatic dysfunction, it should be used with caution to avoid hepatotoxicity.

Hydroxychloroquine: There should be no liberal use of this drug unless prescribed by a registered medical practitioner. This drug is highly toxic and must be weighed against the benefits. This drug prolongs the QT interval in ECG and may cause more harm than good. Elderly persons with cardiac, pulmonary co-morbidities, with diabetes mellitus (type I/II), with metabolic syndromes, especially with COPD and known cardiac problems should avoid this drug. This should be used with caution to the caregivers of corona in hospitals judiciously. HCQ (4- 
Aminoquinoline) acts as a prophylactic drug and therapeutic agent against COVID-19.It acts on host respiratory cell by increasing endodermal $\mathrm{pH}$ required for the virus host cell fusion, increase in $\mathrm{pH}$ disrupts viral functions. HCQ interferes with glycosation of cellular receptors of virus. Chloroquine results in disablement of ACE-2 terminal glycosylation resulting in disruption in the association of COVID-19 and host cell as COVID-19 requires ACE- 2 receptors to attach to the host cell. HCQ acts as an ionophoric agent for zinc ions for cytoplasmic influx. The mechanism of disruption of virus-host cell fusion helps to use HCQ in COVID-19 in periinfective period and effective in the of COVID-19 pneumonia.This HCQ need to be used with caution in G-6-PD efficiency and ocular comorbidities. To summarise 4Aminoquinolines(Chloroquine and $\mathrm{Hy}$ droxychloroquine) act by changing $\mathrm{pH}$ of endosomes.prevents viral entry-transport and postentry events.

\section{Anti-Virals in Covid-19}

There is no specific antiviral treatment for COVID-19.Antivirals like Remdesivir (5), Ribavirin cosmostat mesylate, Damnavir, Umifenovir and beta interferon, aerosolicised alpha interferon, Oseltamivir, Baloxivir, Marboxil are tried with variable benefits. They inhibit viral application to host cells or inhibits viral RNA synthesis and m-RNA capping, inhibits viral replication and/or blocks viral entry and maturation. Favipiravir inhibits viral RNA dependent polymerase. Interferons stimulate innate and viral immunity and inhibits viral replication along with anti-virals like Lopinavir or Ritonavir. Monoclonal antibodies like Sarilumab \& Eculizumab inhibit IL-6 and blocks cytokine storm in full blown picture of COVID-19 Pneumonia with severe respiratory distress. Convalescent plasma (from persons who have recovered from COVID19) contains anti-COVID-19 antibodies. This component from plasma may be used to reduce the severity of disease in COVID-19 positive patients, reducing the viral load by using this plasma containing antibody. Donor \& Patient must have compatible blood groups \& should be screened for serology of HIV, HBSAg, HCV etc.

\section{Clinical Management}

Fever Clinic: To screen fever cases, cases with respiratory infection and difficulty, to separate cases for quarantine, isolation, critical care ICU, sputum and blood tests should be liberalized, mainly APCR. Sorting out of cases is done in fever clinics and are sent to the wards accordingly.

Quarantine: Two types, Home Quarantine (14 days) and Hospital Quarantine (for observation). Home quarantines are given to asymptomatic persons with a history of contact with COVID-19 patients and exposed to persons with a history of travelling abroad. Hospital quarantine is for those who are having symptoms, tests sent and frontline workers to corona positive cases.

Isolation: Admission in hospital and treatment and watching progress and treating accordingly. Tests are done. When the test is positive with aggravation of symptoms, shifted to critical care for active management.

Critical care: In the ICU with round the clock supportive care and medicines, respiratory support with ventilators.

\section{Future Implications}

Future impact of this global corona attack is farreaching and detrimental not only in health sector but also in world-economy largely. In different part of the globe it may come down to the base level so far the infectivity is concerned, but the virus is not extinct globally. There will be some degree of health-hazard in terms of some residual co-morbidities caused by the virus worldwide. Low grade virulence may not draw attention of flu-like symptoms but its infectivity will perpetuate the viral existence within the globe with occasional resurgence as pandemic. Total loss of active working manpower will cripple the world-economy. Thousands will be jobless with hunger, disease and lacklustre confidence. Psychological turmoil will be the allpervading situation.

Along with corona pandemic there is loss of work, millions of jobless workers in India are compelled to return back hungry and moneyless to wards, hometown or village. This lockdown compels them to stay in roads, bus stands, stations, buses and trucks. They are staying in temporary shelters \& tents, half-fed or no food in quarantine. This economic pandemic will shift us downward with disaster, deaths, economic shutdown and low GDP unless quick measures are taken by the government to boost the economy of the poorest of the poor workers. There will be world-wide economic disaster for pro- 
longation of lockdown devastating all round economy structure. Crores of people will be jobless, mainly in unorganised sectors. The socioeconomic infrastructure of poverty stricken, hungry and sudden workless people migrating from workplace to the hometown and villages need to be sheltered, given food and arrangement for quarantine and arranged transport in due course.

A vast majority of people will be pushed down below poverty line. Chronic health hazards of the sufferers, survivors and carriers (both symptomatic and asymptomatic) will increase all round morbidity and mortality, thus further dampening the economic pandemic. Hope workers worldwide withstand against this deadly virus and will come up with flying colours in near future. Let us hope for the best that this pandemic will cool down. COVID-19 is not biding goodbye There will be carriers spread all over the society, if not globally. So the hygienic habits, the contamination chain breakage, social distancing, use of mask, lifestyle of modifications to reduce the co-morbidities has to be practiced socially throughout. Respiratory care \& exercise, avoidance of smoking, tobacco and alcohol should be practiced with saturning discipline.

In national perspective, our India is a vast mixed population with different levels of education, consciousness, economy and socialsituation with poverty, ignorance and lacklustre social awareness. Even the qualified, conscious people are taking everything casually in all the events of janta curfew, lockdown, social distancing and personal hygiene. Our social consciousness need to be elevated as it is a prerequisite of our existence. This is great lesson we learnt from COVID-19 crisis which shook the globe recently. For a future, we have to fight, be disciplined and maintain ecosystem of this planet in harness for survival of the fittest as human being.

\section{References}

1. Abduljalil JM, Abduljalil BM. Epidemiology, genome, and clinical features of the pandemic SARS-CoV-2: a recent view. New Microbes New Infect.2020 Mar 31;35:100672. doi: 10.1016/j.nmni.2020.100672.

2. https://www.icmr.nic.in/content/covid-19. 3.https://www.who.int/emergencies/diseases/novel -coronavirus-2019/technical-guidance.

4.Choudhary R, Sharma AK, Choudhary R. Potential use of hydroxychloroquine, ivermectin and azithromycin drugs in fighting COVID-19: trends, scope and relevance. New Microbes New Infect. 2020 Apr 22:100684. doi: 10.1016/j.nmni.2020.100684.

5. Cao YC, Deng QX, Dai SX. Remdesivir for severe acute respiratory syndrome coronavirus 2 causing COVID-19: An evaluation of the evidence. Travel Med Infect Dis. 2020 Apr 2:101647. doi: 10.1016/j.tmaid.2020.101647. 